\title{
The headache under-response to treatment (HURT) questionnaire, an outcome measure to guide follow-up in primary care: development, psychometric evaluation and assessment of utility
}

\author{
T. J. Steiner ${ }^{1,2^{*}}$, D. C. Buse ${ }^{3,4}$, M. Al Jumah ${ }^{5}$, M. L. Westergaard ${ }^{6}$, R. H. Jensen ${ }^{6}$, M. L. Reed ${ }^{7}$, L. Prilipko ${ }^{8^{\wedge}}$, \\ F. S. Mennini ${ }^{9,10}$, M. J. A. Láinez ${ }^{11}$, K. Ravishankar ${ }^{12,13}$, F. Sakai ${ }^{14}$, S.-Y. Yu ${ }^{15}$, M. Fontebasso ${ }^{16}$, A. Al Khathami ${ }^{5}$, \\ E. A. MacGregor ${ }^{17}$, F. Antonaci ${ }^{18,19}$, C. Tassorelli ${ }^{16,19}$, R. B. Lipton ${ }^{3,4}$ and on behalf of Lifting The Burden: The Global \\ Campaign against Headache
}

\begin{abstract}
Background: Headache disorders are both common and burdensome but, given the many people affected, provision of health care to all is challenging. Structured headache services based in primary care are the most efficient, equitable and cost-effective solution but place responsibility for managing most patients on health-care providers with limited training in headache care. The development of practical management aids for primary care is therefore a purpose of the Global Campaign against Headache. This manuscript presents an outcome measure, the Headache Under-Response to Treatment (HURT) questionnaire, describing its purpose, development, psychometric evaluation and assessment for clinical utility. The objective was a simple-to-use instrument that would both assess outcome and provide guidance to improving outcome, having utility across the range of headache disorders, across clinical settings and across countries and cultures.

Methods: After literature review, an expert consensus group drawn from all six world regions formulated HURT through item development and item reduction using item-response theory. Using the American Migraine Prevalence and Prevention Study's general-population respondent panel, two mailed surveys assessed the psychometric properties of HURT, comparing it with other instruments as external validators. Reliability was assessed in patients in two culturally-contrasting clinical settings: headache specialist centres in Europe $(n=159)$ and primary-care centres in Saudi Arabia $(n=40)$. Clinical utility was assessed in similar settings (Europe $n=201$; Saudi Arabia $n=342$ ).

(Continued on next page)
\end{abstract}

\footnotetext{
* Correspondence: t.steiner@imperial.ac.uk

Deceased

${ }^{1}$ Department of Neuromedicine and Movement Science, NTNU Norwegian

University of Science and Technology, NO-7941 Trondheim, Norway

2Division of Brain Sciences, Imperial College London, London, UK

Full list of author information is available at the end of the article
} 
(Continued from previous page)

Results: The final instrument, an 8-item self-administered questionnaire, addressed headache frequency, disability, medication use and effect, patients' perceptions of headache "control" and their understanding of their diagnoses. Psychometric evaluation revealed a two-factor model (headache frequency, disability and medication use; and medication efficacy and headache control), with scale properties apparently stable across disorders and correlating well and in the expected directions with external validators. The literature review found few instruments linking assessment to clinical advice or suggested actions: HURT appeared to fill this gap. In European specialist care, it showed utility as an outcome measure across headache disorders. In Saudi Arabian primary care, HURT (translated into Arabic) was reliable and responsive to clinical change.

Conclusions: With demonstrated validity and clinical utility across disorders, cultures and settings, HURT is available for clinical and research purposes.

Keywords: Headache disorders, Migraine, Tension-type headache, Cluster headache, Medication-overuse headache, Management, Treatment planning, Treatment optimization, Acute treatment, Preventative treatment, Treatment satisfaction, Assessment, Burden, Instruments, Outcome measures, HURT questionnaire, Follow-up, Primary care, Global campaign against headache,

\section{Background}

Headache disorders are very common, affecting half or more of all adults and many children worldwide [1], and they are highly burdensome [2-8]. The World Health Organization (WHO) recognised them nearly 20 years ago as a global public-health priority [9]. Among them, migraine and tension-type headache (TTH) are often lifelong illnesses. Medication-overuse headache ( $\mathrm{MOH})$, usually a complication of migraine or TTH caused by mistreatment of one or the other, is highly frequent, often present daily or almost so. These three headache disorders are the substantial contributors to public ill health, although there are many others [10]. They cause pain, hinder work, damage family and social relationships and impoverish quality of life (QoL).

Headache disorders are largely treatable [11], and they demand treatment not only because it is inhumane to ignore these burdens of ill health. The high levels of disability associated with headache [2-8] lead to very large productivity losses, so that these disorders are very costly in a financial sense $[12,13]$. Although the economic consequences of improving headache care are not well studied empirically [14], health services that make the effective remedies more widely available would very probably be cost saving in most economies [12, 15]. Treatment of headache is therefore demanded also because it is economically sensible.

Nevertheless, given the very large numbers of people affected, adequate provision of health care to everyone for whom self-care is insufficient - perhaps $50 \%$ of those with a headache disorder [16] - is highly challenging.

Structured headache services, with their basis in primary care and supported by educational initiatives, are the most efficient, equitable and cost-effective solution [12]. Such services place responsibility for managing most people with headache on health-care providers who lack special expertise in headache management [16]. This is not in itself problematic: medical management of headache disorders, for the great majority of people affected by them, requires no specialist expertise or investigations but only the diagnostic and management skills that are available to all physicians and many other health-care providers in primary care $[12,16]$. On the other hand, non-specialists throughout the world may have received limited training in the application of these basic skills to headache diagnosis and treatment [12].

The development of practical management aids for primary care therefore became an early objective of the Global Campaign against Headache [17-19], a programme of action for the benefit of people with headache conducted by the UK-registered nongovernmental organization Lifting The Burden (LTB) in official relations with WHO [20]. This manuscript presents one such aid, the Headache Under-Response to Treatment (HURT) questionnaire; it describes its purpose and its development, and summarises the studies undertaking its psychometric evaluation and assessment for clinical utility.

\section{Objective}

We believed that a simple outcome measure was insufficient. Whenever treatment of a patient is started, or changed, follow-up should either ensure that treatment is optimal or recognise that it is not and identify the change(s) in treatment needed. Evaluation of outcome should therefore be coupled with guidance to improve outcome. While this was the instrument's perceived purpose, it is not so simple a purpose - resources, services and expectations vary greatly between countries and 
cultures - and no other treatment-assessment instrument met it [21]. No previously described instrument had been shown to function for all important headache disorders or across cultures, and no single instrument covered the range of assessment and decision-making necessary for successful headache management [21]. Few made the key link between assessment and clinical advice, essential because, even in optimal circumstances, outcomes are rarely perfect. Judgement may be needed as to whether the outcome achieved in an individual patient is the best that the patient can reasonably expect. For the non-specialist in particular, one question sometimes arising is: "What further effort, in hope of a better outcome, is justified?" A second question, which may follow, is "What is it that needs changing?"

The objective, therefore, was an instrument that would both assess outcome and provide answers to these two questions, offering guidance on appropriate actions towards treatment optimisation. More specifically, we aimed to create a short, simple-to-use management aid, versatile yet reliable and sensitive, which met the purpose described and three utility requirements:

1. in both primary and specialist care;

2. across the range of headache disorders of publichealth importance;

3. across countries and cultures, despite wide variation in resources, services and expectations.

This objective would be reached through a development process invoking expert consensus followed by psychometric and clinical evaluations involving professional and lay representatives of all world regions.

\section{Methods}

Development was a multi-stage process including item development, item reduction using item-response theory (IRT), psychometric testing and external validation, and assessment of clinical utility.

For item development, LTB brought together an international expert consensus group (Table 1), with the technical support of WHO. The members, from all six world regions, included headache specialist health-care professionals, primary-care physicians, lay patient advocates and experts in health economics, psychometrics and qualitative research. The group met first in April 2006 at WHO headquarters in Geneva and agreed the domains that the instrument should address. After a literature review of existing instruments with related purposes, a large item pool within these domains was developed. In further discussions within the group, and through consensus, initial item selection was followed by a process of item reduction, refining the selection and
Table 1 Development group

Convener: TJ Steiner (UK)

Meeting in Geneva

RB Lipton (USA) (chairman), M Al Jumah (Saudi Arabia), B Eggleston (Australia) (lay), M Fontebasso (UK), L Gardella (Argentina), RH Jensen (Denmark), H Kettinen (Finland) (lay), MJA Láinez (Spain), FS Mennini (Italy), M Peters (UK), L Prilipko (WHO), K Ravishankar (India), F Sakai

(Japan), N Sharma (India) (lay), TJ Steiner (UK), AD Tehindrazanarivelo (Madagascar), D Valade (France), S-Y Yu (China)

Contributing remotely

W Stewart (USA), M Von Korff (USA)

Meeting in New York

RB Lipton (USA) (chairman), M Al Jumah (Saudi Arabia), S Broner (USA),

DC Buse (USA), ML Reed, (USA), D Serrano (USA), M Fontebasso (UK), FS Mennini (Italy), TJ Steiner (UK)

Contributing remotely

$\mathrm{RH}$ Jensen (Denmark)

limiting the number of items to those considered most apt (relevant and informative). These candidate items were formulated into simple questions, with response options based on the group's expert knowledge and clinical experience, and included in the emerging instrument. A scoring system was constructed around them, with guidance on interpretation. Accompanying clinical advice, according to responses, was developed through reference to authoritative treatment guidelines from multiple countries coupled again with the group's expert input. A design process then built a first version ("HURT-v1") of the end-product as a 2-page document.

The Geneva group's final task was to name the instrument.

Psychometric evaluation (see below) was carried out on HURT-v1. Informed by the results, a smaller group met in New York City in October 2007 (Table 1), making changes as necessary, again through consensus, and generating the final version ("HURT-final"). Clinical evaluations were performed on HURT-final.

\section{Evaluation}

Two groups were involved in evaluation of HURT, one in psychometric testing and the other in clinical assessments (Table 2).

\section{Psychometric testing and validation}

The American Migraine Prevalence and Prevention (AMPP) Study [22] recruited from a panel constructed and maintained by the survey company NFO, Inc. (Ames, IA, USA) [23] to match the US population, according to the national census, by age, gender, socioeconomic status and region of the country. The AMPP screening survey identified participants describing themselves as affected by "severe headache", a term intended preferentially to capture those with migraine and/or with unmet needs for headache treatment. We included 
Table 2 Evaluation groups

Psychometric evaluation

M Al Jumah (Saudi Arabia), A Al Khathami (Saudi Arabia),

A Al Owayed (Saudi Arabia), DC Buse (USA), A Jawhary (Saudi Arabia),

$\mathrm{RH}$ Jensen (Denmark), S Kojan (Saudi Arabia), RB Lipton (USA), EA

MacGregor (UK), ML Reed (USA), D Serrano (USA), CM Sollars (USA), TJ

Steiner (UK), H Tamim (Saudi Arabia), ML Westergaard

(Philippines/Denmark)

Clinical evaluation

M Al Jumah (Saudi Arabia), A Al Khathami (Saudi Arabia), A Al Owayed (Saudi Arabia), F Antonaci (Italy), DC Buse (USA), A Jawhary

(Saudi Arabia), RH Jensen (Denmark), S Kojan (Saudi Arabia), RB Lipton (USA), EA MacGregor (UK), TJ Steiner (UK), H Tamim (Saudi Arabia), C

Tassorelli (Italy), ML Westergaard (Philippines/Denmark)

HURT-v1 in two surveys mailed to samples of these AMPP Study participants.

\section{Ethics approval}

The AMPP Study, with inclusion of HURT, was approved by the Albert Einstein College of Medicine Institutional Review Board. The mailings included explanations of the study, and voluntary participation in the surveys implied consent.

\section{Sociodemographic variables}

Recorded in these surveys were age, gender, weight and height, all self-reported. Body-mass index (BMI) was calculated from height and weight using the WHO standard formula. Data were also collected on average annual household income, race and geographic region.

\section{US mailing \#1: Psychometric testing}

HURT-v1, including all candidate items and response options, was posted to a first sample $(N=2500)$ in summer, 2006. Enclosed in the mailing were question sets on the sociodemographic variables and on headache frequency, severity, associated symptoms and treatment.

The headache-related data were used to diagnose participants with migraine, using the American Migraine Study (AMS)/AMPP Study diagnostic module (demonstrated to have $100 \%$ sensitivity and $82 \%$ specificity for this diagnosis) [24]. Case-definition criteria for migraine accorded with ICHD-II [25], except that a lifetime history of $\geq 5$ attacks could not be ascertained from the cross-sectional data. No significant changes have occurred between ICHD-II [25] and ICHD-3-beta [10] with regard to these criteria. Only participants with headache on $\leq 14$ days/month were included as migraine cases (ie, episodic migraine) in this analysis.

\section{Item analysis}

Responses from these participants to HURT items 1-6 were included in a graded item-response model applied to assess scale properties using SAS NLMIXED and to identify an underlying latent variable. However, the
HURT metrics for items 1-6 varied: four were count items (with two based on 1 month's recall and two based on 3 months' recall) and two were polytomous response items. To simplify item analysis, we harmonized item responses, placing all on an identical metric. Count items were converted to five-category polytomous items, with severity values in the range $0-4$ as follows:

1. 0 (very mild): 0 days in 1 month or 0 days in 3 months;

2. 1 (mild): $1-2$ days in 1 month or $1-5$ days in 3 months;

3. 2 (moderate): $3-5$ days in 1 month or 6-10 days in 3 months;

4. 3 (severe): 6-15 days in 1 month or 11-20 days in 3 months;

5. 4 (very severe): $\geq 16$ days in 1 month or $\geq 21$ days in 3 months.

The original two polytomous response items were harmonized so that higher-ordered response options were also indicative of greater severity. Consequently, the medication efficacy item was reverse-coded (0-4) to reflect increasing inefficacy, and the headache-control item was reverse-coded (0-4) to reflect increasing lack of control. Total and factor-specific scores could then be computed as simple sums of the scale items.

An item-response model was fitted to these item scores using a multinomial response-distribution. Item parameters were estimated for the five response categories offered for each item. A confirmatory factor analysis model was also employed where the original count items loaded on the first factor and the original polytomous items loaded on the second. The correlation between the two factors was freely estimated.

\section{Performance across headache types}

In the second mailing sample (described below), we attempted to contrast HURT factor scores across headache types to assess relative performance.

\section{US mailing \#2: External validity}

HURT-v1 was posted, in autumn 2006, to a different sample $(N=2250)$, along with similar sociodemographic and headache question sets. Included in this mailing were multiple external validators: other instruments used in headache management and themselves wellvalidated as measures of headache-attributed impact, treatment optimization, health-related QoL (HRQOL) or comorbid depression. These are described in Table 3. For purposes of correlation with these external validators, we used the total HURT scores derived during item 
Table 3 Instruments used as external validators

\begin{tabular}{|c|c|c|}
\hline Instrument & Structure/purpose & Scoring \\
\hline $\begin{array}{l}\text { Migraine Disability } \\
\text { Assessment (MIDAS) } \\
\text { scale [29] }\end{array}$ & $\begin{array}{l}5 \text { items counting days of lost productivity } \\
\text { (complete or by }>50 \%) \text { from paid or household } \\
\text { work and missed family, social or leisure activities } \\
\text { during the preceding } 3 \text { months }\end{array}$ & $\begin{array}{l}\text { Sum of responses (the MIDAS score) } \\
\text { quantifies impact in days lost per } 3 \text { months }\end{array}$ \\
\hline $\begin{array}{l}\text { Headache Impact Test } \\
\text { (HIT-6) [30] }\end{array}$ & $\begin{array}{l}6 \text { items assessing lost time at work, school or social } \\
\text { activities, pain severity, fatigue, frustration and difficulty } \\
\text { in concentration, each with } 5 \text { frequency response } \\
\text { options (never, rarely, sometimes, very often, always) }\end{array}$ & $\begin{array}{l}\text { Response options scored 6, 8, 10, } 11 \\
\text { and } 13 \text { respectively, with higher summed } \\
\text { scores (range 36-78) indicating } \\
\text { greater impact }\end{array}$ \\
\hline $\begin{array}{l}\text { Migraine Specific Quality } \\
\text { of Life (MSQ) questionnaire, } \\
\text { version } 2.1[32]\end{array}$ & $\begin{array}{l}14 \text { items assessing QoL in the preceding } 4 \text { weeks in } 3 \\
\text { migraine-specific dimensions (role function-restrictive; } \\
\text { role function-preventive; emotional function), each } \\
\text { item having } 6 \text { Likert-type response options. }\end{array}$ & $\begin{array}{l}\text { On a scale } 0-100 \text { (higher being better QoL) } \\
\text { through transformation involving } 3 \text { steps }\end{array}$ \\
\hline $\begin{array}{l}\text { PRIME-MD Patient Health } \\
\text { Questionnaire - depression } \\
\text { module (PHQ-9) [31] }\end{array}$ & $\begin{array}{l}\text { Depressive symptoms over the preceding } 2 \text { weeks } \\
\text { assessed in the } 9 \text { domains of DSM-IV, each with } 4 \\
\text { frequency response options (not at all, on several } \\
\text { days, on more than half of days, nearly every day) }\end{array}$ & $\begin{array}{l}\text { Response options scored } 0-3 \text {, with higher } \\
\text { summed scores (range } 0-27 \text { ) indicating more } \\
\text { severe depression }\end{array}$ \\
\hline $\begin{array}{l}\text { Migraine Prevention } \\
\text { Questionnaire (MPQ) [33] }\end{array}$ & $\begin{array}{l}5 \text { items (regarding headache frequency, acute } \\
\text { medication use and headache-related impairment, } \\
\text { worry and anxiety) identify need for, and guide, } \\
\text { preventative pharmacological treatment of } \\
\text { migraine based on consensus guidelines }\end{array}$ & $\begin{array}{l}\text { Responses summed into a total score, which } \\
\text { falls into one of } 3 \text { categories: preventative } \\
\text { treatment not indicated, should be } \\
\text { considered or should be offered }\end{array}$ \\
\hline
\end{tabular}

analysis (see above). Comparisons employed Spearman correlations.

\section{Reliability}

Two studies were undertaken to assess reliability, in very different cultural and clinical settings. Both have been published previously [26, 27], and are therefore only summarised here.

HURT-final was completed twice by 159 consecutive patients seeking non-urgent care in headache specialist centres in Denmark and United Kingdom (UK) [26]. The instrument was first mailed to patients as they joined the clinic waiting lists, before their initial visits, and then given to them at these initial visits, which were expected usually to take place about 1 month later.

For testing in Arabic-speaking patients in Saudi Arabia, HURT was translated according to LTB's translation protocol for hybrid documents [28]. Test-retest reliability was assessed in 40 consecutive patients of four primary-care centres, who completed HURT at two visits 4-6 weeks apart while receiving usual care [27].

\section{Ethics approval}

In Denmark and UK, evaluation of HURT as an outcome measure was considered a service-improvement project, falling outside the scope of research ethics review [26]. Ethics approval was requested in Saudi Arabia, and granted by the Institutional Review Board of National Guard Health Affairs [27]. In both studies, information was provided and all participants gave consent.

\section{Clinical testing}

The first assessment of clinical utility, to demonstrate that HURT was responsive to change induced by effective management, was conducted in headache specialist centres in three European countries [26]. In Denmark and UK, HURT was administered on a third occasion to the same 159 consecutive patients when, in each case, the treating specialist judged that the best possible outcome had been achieved. In Italy, HURT was answered by 42 patients at initial and final visits [26].

\section{Clinical testing}

The second assessment evaluated the Arabic version of HURT in primary care in Saudi Arabia. The general practitioners (GPs) in four centres were trained in headache management, and the centres then randomized in pairs to control (standard care) or intervention (care guided by implementation of HURT). Responsiveness of HURT to clinical change was assessed by comparing base-line responses to HURT questions 1-6 with those at follow up. Clinical utility was assessed by comparing outcomes between control and intervention pairs after 3 months, using locally-developed 5-point verbal-rating scales: patient-satisfaction scale (PSS) and doctorsatisfaction scale (DSS) [27].

\section{Ethics approval}

In Denmark and UK, these studies again fell outside the scope of research ethics review [26]. Ethics approvals were obtained in Italy from the ethics review committee of C Mondino Foundation, University of Pavia [26], and in Saudi Arabia from the Institutional Review Board of the King Abdullah International Medical Research 
Center (KAIMRC) [27]. In both studies, information was provided and all participants gave consent.

\section{Results}

The Geneva meeting identified the following as essential domains: headache frequency, disability, medication use and effect, patients' perceptions of headache "control" and their understanding of their diagnoses. The group developed an 8-item self-administered questionnaire addressing these. Responses were either numerated in days over a one- or three-month recall-period or selected from Likert options. In either case, responses were graded into an area of "no concern" or one of three flagged areas indicating increasingly important treatment deficiencies; clinical advice was provided for each of the latter.

The design process constructed the questionnaire, with responses, interpretation and advice, into the form shown (Additional file 1).

After the psychometric evaluations, and informed by them, the New York meeting made only minimal changes to generate HURT-final.

\section{Psychometric evaluation \\ Psychometric testing}

In the assessment of scale properties, valid returns came from 1691 (68\%) respondents to the first US mailing. A large majority (1362; 81\%) met ICHD-II criteria for migraine [25]. IRT revealed a two-factor model: 1) headache frequency, disability and medication use; and 2) medication efficacy and headache control items. Total and sub-factor scores correlated strongly (Spearman coefficient $r=0.49-0.86$ ).

\section{Performance across headache types}

In the second US mailing sample, which, like the first, were self-selecting for "severe" headache, sub-sample sizes for headache types other than episodic migraine were very low $(\leq 100)$ : 73 cases met criteria for episodic TTH (ETTH); 100 had headache on $\geq 15$ days/month, with ten of these diagnosed as chronic migraine. Several simplifying modifications were attempted in order to contrast other headache types with episodic migraine, but all models failed in the face of these small samples and insufficient information. Furthermore, respondents with headache on $\geq 15$ days/month inevitably reported a constant (maximal) value on HURT item 1 (headache days/month). As a last resort, fitting the item-response model separately to the episodic migraine sub-sample and the headache on $\geq 15$ days/month sub-sample (the only other for which the model contrast was estimable), we compared the estimated factor scores for these two groups in an ANOVA (Table 4). No significant differences were observed in factor scores for the two factors, suggesting no meaningful difference in the performance of HURT between these headache types.

\section{External validity}

Valid returns to the second mailing were received from 1734 (69\%) respondents, of whom 1391 (80\%) met ICHD-II criteria for migraine [25]. Summed HURT scores correlated in the expected directions with the other measures. Correlations were positive with both the MIDAS questionnaire $(r=0.69)$ and HIT-6 $(r=0.49)$, instruments that are strongly influenced by disability $[29,30]$, and with PHQ-9 $(r=0.34)$, a measure of depression [31]. Correlations were negative with MSQ version 2.1, a migraine-specific QoL instrument [32], so that higher HURT scores were associated with poorer QoL (restrictive subscale: $\mathrm{r}=-0.53$; preventative subscale: $\mathrm{r}=$ -0.58 ; emotional subscale: $r=-0.51$ ). HURT scores correlated most strongly, and positively, with MPQ $(r=$ 0.86), an instrument indicating need for preventative pharmacological treatment of migraine [33]. These correlations suggested that, while the instruments shared some variance, they nonetheless captured unique constructs.

\section{Reliability}

Test-retest reliability in specialist care in Denmark and UK was fair to low ( $\kappa=0.38-0.62 ; r=0.49-0.76)$ [26]. It should be noted that, while the interval between first and second applications of HURT was intended in the study design to be about 1 month, subsequent review of the Danish records revealed a range between 1 day and 9 months (median 1.7 months). The longer-thanplanned intervals were caused in part by the centre's extending waiting time but also in part by patients who changed appointment dates - sometimes introducing long delays. Internal consistency reliability on the other hand was very good (Cronbach's $\alpha=0.85$ ) [26]. In Saudi Arabian primary care, intra-class correlation coefficients were $0.66-0.78$ (all $p \leq 0.001$ ) for questions $1-4$ and $0.90-0.93$ for questions $5-7$ (all $p<0.0001$ ) [27]. For the dichotomous response to question $8, \mathrm{k}=1(\mathrm{p}<0.0001)$. Internal consistency reliability was good (Cronbach's $\alpha=$ 0.74) [27].

Table 4 Estimated factor scores for episodic migraine subsample versus headache on $\geq 15$ days/month sub-sample

\begin{tabular}{llllll}
\hline Source & DF & $\begin{array}{l}\text { Type III sum } \\
\text { of squares }\end{array}$ & $\begin{array}{l}\text { Mean } \\
\text { square }\end{array}$ & $F$ & $\operatorname{Pr}>F$ \\
\hline Migraine & 1 & 0.5951 & 0.5951 & 0.78 & 0.3763 \\
Effect & 1 & 0.1050 & 0.1050 & 0.14 & 0.7101 \\
Migraine*Effect & 1 & 0.1579 & 0.1579 & 0.21 & 0.6486 \\
\hline
\end{tabular}




\section{Clinical evaluation}

In specialist-care utility evaluation in 201 patients in Europe, with HURT administered at the start and end of treatment, good outcomes were judged to have been achieved in 155 (77\%) [26]. HURT reflected these in significant differences between recorded responses at base line and postintervention $(p<0.01)$, indicating responsiveness of the instrument to change. Analyses of paired responses (before and after treatment) found seven of the eight HURT items indicating improvements $(p<0.001)$. No evident improvement in patients' concerns about side-effects of medication was indicated by item 7 ( $p=0.18)$ [26].

In 342 patients in Saudi Arabian primary care, HURT signalled clinical improvement over 3 months through statistically significant reductions $(p<0.0001)$ in responses to each of questions 1-6 [27]. HURT also distinguished clearly between patients with good or poor outcomes, with questions $1-4$ each $(\mathrm{p}<0.0001)$ indicating improvement in those reporting satisfaction (positive PSS scores) and worsening (or no improvement) in those reporting dissatisfaction (negative PSS scores) [27]. There was a trend $(p=0.06)$ towards greater patientsatisfaction among those treated with guidance from HURT $(n=207)$ than among controls $(n=135)$. There was a slight opposite trend in doctor-satisfaction [27].

\section{Discussion}

HURT is a consensus-developed 8-item self-administered questionnaire addressing headache frequency, disability, medication use and effect, patients' perceptions of headache "control" and their understanding of their diagnoses. Its scope is unique among outcome measures.

In the evaluations, HURT proved to be a psychometrically sound instrument for monitoring headache treatment, with, importantly, scale properties apparently stable across headache disorders. While test-retest reliability in specialist care was assessed as fair to low, this was, very probably, because headache in this setting, and prior to start of management, was unstable over time. Although this was not intended, intervals of up to 9 months between the two applications of HURT allowed the possibility of real change in many patients. In the very different setting of Saudi Arabian primary care, reliability was good for questions 5 to 7 , to which responses expressed beliefs or perceptions at the time of testing. It was less good for questions 1 to 4 , which required recall of past events over 1 to 3 months and which, again, were subject to real changes in the frequencies of events potentially occurring over the testretest period. It should be noted also that, unlike in Europe, patients in Saudi Arabia did receive an intervention (albeit only standard care) between the two administrations of HURT. The two settings were, therefore, not comparable for testing reliability (it was not intended that they should be). In USA, summed scores correlated well and in the expected directions with external validation measures.

The literature search yielded a multiplicity of psychometrically robust instruments available to support the several key steps in comprehensive care of headache disorders [21]. These instruments can be used to aid the diagnosis of primary headache disorders; the exclusion of secondary headaches; the assessment of headache symptoms and their severity, headache-attributed disability, burden and impact (both ictal and interictal); the recognition and assessment of comorbidities, and of psychological factors shown to predict adherence to treatment and outcomes; outcome measurement including patients' satisfaction; and treatment planning, monitoring and optimization for both acute and preventative pharmacological therapies. Many have particular strengths. MIDAS in particular, although it measures headache-attributed lost time rather than disability [34], is scored in meaningful units, is easy to use, has demonstrated reliability and validity, predicts treatment needs, has been extensively used in clinical practice in many countries and is sensitive to change, at least in severelyaffected patients. However, no single instrument other than HURT combines so many of these aspects of care, and, especially, few instruments link assessment to clinical advice or suggested actions [21]. Thus, they do not serve non-headache experts well, especially health-care providers in primary care.

The HURT questionnaire was created to fill this gap and it appears to have the capability of doing so.

In Europe, HURT showed utility in specialist care as an outcome measure across headache disorders. In this setting, where many patients are partially or wholly refractory even to best care, HURT can be used to define and explain treatment goals. In addition, it can promote self-efficacy and knowledge about headache. In Saudi Arabian primary care, where no other Arabic instruments were available as standards for comparison, HURT was reliable and responsive to clinical change and therefore it has clinical utility in Arabic-speaking patients. One explanation of the apparent discord between doctors' and patients' satisfaction in Saudi Arabia is that the instrument worked as it should - improving care (perceived by patients) while revealing deficiencies in care and need for improvement to the physicians.

\section{Conclusions}

The HURT questionnaire has demonstrated validity and clinical utility, and is currently available for clinical use and research purposes in English and Arabic. Some other translations are known to exist, and future work may include use and evaluation in other countries and cultures. 


\section{Additional file}

Additional file 1: HURT questionnaire. (PDF $105 \mathrm{~kb}$ )

\section{Abbreviations}

AMPP: American Migraine Prevalence and Prevention Study; ANOVA: analysis of variance; DSS: doctor-satisfaction scale; GP: general practitioner; HIT6: Headache Impact Test; HURT: Headache Under-Response to Treatment; ICHD: International Classification of Headache Disorders; IRT: item-response theory; LTB: Lifting The Burden; MIDAS: Migraine Disability Assessment scale; $\mathrm{MOH}$ : medication-overuse headache; MPQ: Migraine Prevention Questionnaire; MSQ: Migraine Specific Quality of Life questionnaire; PHQ: PRIME-MD Patient Health Questionnaire; PSS: patient-satisfaction scale; QoL: quality of life; TTH: tension-type headache; UK: United Kindom; USA: United States of America; WHO: World Health Organization

\section{Acknowledgements}

We are grateful to Susan Broner (USA), Beth Eggleston (Australia), Lorenzo Gardella (Argentina), Hilkka Kettinen (Finland), Michele Peters (UK), Daniel Serrano (USA), Neeha Sharma (India), C Mark Sollars (USA), Walter Stewart (USA), Alain Tehindrazanarivelo (Madagascar/France), Dominique Valade (France) and Michael Von Korff (USA) for intellectual input at various stages of development of the HURT questionnaire.

\section{Funding}

Financial support for the consensus meetings in Geneva and New York was provided by Lifting The Burden. The AMPP study was funded originally by a grant from Ortho-McNeil Pharmaceuticals to the National Headache Foundation (USA), and later supported by several pharmaceutical companies collectively. While the AMPP included our mailings, these received no specific support. There was no other external funding for the studies or analyses reported here. Lifting The Burden determined the purpose and objectives of the HURT questionnaire as a product of the Global Campaign against Headache, but there were no other influences upon these studies from funding sources.

\section{Availability of data and materials}

Not applicable.

\section{Authors' contributions}

TJS, DCB, MAJ, RHJ, MLR LP, FSM, MJAL, KR, FS, SYY, MF and RBL were contributors to the consensus meetings in Geneva and/or New York. MAJ, AAK, DCB, RHJ, RBL, EAM, MLR, TJS and MLW contributed to design, implementation and/or analysis of the psychometric evaluation studies. MAJ, AAK, FA, DCB, RHJ, RBL, EAM, TJS, CT and MLW contributed to design, implementation and/or analysis of the clinical evaluation studies. TJS and DCB drafted the manuscript. All authors contributed to revision of the manuscript and approved the final version

\section{Ethical approval and consent to participate}

Stated in text.

\section{Consent for publication}

Not applicable.

\section{Competing interests}

TJ Steiner and RH Jensen are Directors and Trustees of Lifting The Burden. RB Lipton, DC Buse and ML Reed are collaborators in the American Migraine Prevalence and Prevention Study (AMPP) study. None of the authors otherwise declares competing interests in relation to the content of this manuscript.

\section{Publisher's Note}

Springer Nature remains neutral with regard to jurisdictional claims in published maps and institutional affiliations.

\section{Author details}

'Department of Neuromedicine and Movement Science, NTNU Norwegian University of Science and Technology, NO-7941 Trondheim, Norway.

${ }^{2}$ Division of Brain Sciences, Imperial College London, London, UK.

${ }^{3}$ Department of Neurology, Albert Einstein College of Medicine, Bronx, NY, USA. ${ }^{4}$ Montefiore Headache Center, Montefiore Medical Center, Bronx, NY, USA. ${ }^{5}$ King Abdullah International Medical Research Center, King Saud Bin Abdulaziz University for Health Sciences, National Guard Health Affairs, Riyadh, Saudi Arabia. ${ }^{6}$ Danish Headache Centre, Department of Neurology, Rigshospitalet Glostrup University of Copenhagen, Glostrup, Denmark. ${ }^{7}$ Vedanta Research, Chapel Hill, NC, USA. ${ }^{8}$ Department of Mental Health and Substance Abuse, World Health Organization, Geneva, Switzerland. ${ }^{9} \mathrm{CEIS}$ EEHTA, Faculty of Economics, University of Rome "Tor Vergata", Rome, Italy. ${ }^{10}$ Institute of Leadership and Management in Health, Kingston University, Kingston upon Thames, UK. ${ }^{11}$ Department of Neurology, University Clinic Hospital, Catholic University of Valencia, Valencia, Spain. ${ }^{12}$ The Headache and Migraine Clinic, Jaslok Hospital and Research Centre, Mumbai, India. ${ }^{13}$ Lilavati Hospital and Research Centre, Mumbai, India. ${ }^{14}$ Saitama International Headache Center, Tokyo, Japan. ${ }^{15}$ Department of Neurology, Chinese PLA General Hospital, Bejing, People's Republic of China. ${ }^{16}$ Headache Expert, Author and Headache Education Facilitator, York, UK. ${ }^{17}$ Centre for Neuroscience \& Trauma, Blizard Institute of Cell and Molecular Science, London, UK. ${ }^{18}$ Headache Science Centre, C Mondino National Neurological Institute, Pavia, Italy. ${ }^{19}$ Department of Brain and Behavioural Sciences, University of Pavia, Pavia, Italy.

Received: 8 January 2018 Accepted: 27 January 2018

Published online: 14 February 2018

\section{References}

1. Stovner LJ, Hagen K, Jensen R, Katsarava Z, Lipton RB, Scher Al, Steiner TJ, Zwart J-A (2007) The global burden of headache: a documentation of headache prevalence and disability worldwide. Cephalalgia 27:193-210

2. Vos T, Flaxman AD, Naghavi M, Lozano R, Michaud C, Ezzati M et al (2012) Years lived with disability (YLDs) for 1160 sequelae of 289 diseases and injuries 1990-2010: a systematic analysis for the global burden of disease study 2010. Lancet 380:2163-2196

3. Steiner TJ, Stovner LJ, Birbeck GL (2013) Migraine: the seventh disabler. J Headache Pain 14:1

4. Vos T, Barber RM, Bell B, Bertozzi-Villa A, Biryukov S, Bolliger I et al (2015) Global, regional, and national incidence, prevalence, and years lived with disability for 301 acute and chronic diseases and injuries in 188 countries, 1990-2013: a systematic analysis for the global burden of disease study 2013. Lancet 386:743-800

5. Vos T, Allen C, Arora M, Barber RM, Bhutta ZA, Brown A et al (2016) Global, regional, and national incidence, prevalence, and years lived with disability for 310 diseases and injuries, 1990-2015: a systematic analysis for the global burden of disease study 2015. Lancet 388:1545-1602

6. Steiner TJ, Stovner LJ, Vos T (2016) GBD 2015: migraine is the third cause of disability in under 50s. J Headache Pain 17:104

7. Feigin VL, Abajobir AA, Abate KH, Abd-Allah F, Abdulle AM, Abera SF et al (2017) Global, regional, and national burden of neurological disorders during 1990-2015: a systematic analysis for the global burden of disease study 2015. Lancet-Neurol 16:877-897

8. Vos T, Abajobir AA, Abbafati C, Abbas KM, Abate KH, Abd-Allah F et al (2017) Global, regional, and national incidence, prevalence, and years lived with disability for 328 diseases and injuries for 195 countries, 1990-2016: a systematic analysis for the global burden of disease study 2016. Lancet 390: 1211-1259

9. World Health Organization (2000) Headache disorders and public health. WHO, Geneva

10. Headache Classification Committee of the International Headache Society (2013) The international classification of headache disorders, 3rd edition (beta version). Cephalalgia 33:629-808

11. Steiner $T J$, Paemeleire $K$, Jensen $R$, Valade $D$, Savi $L$, Lainez MJA, Diener $\mathrm{H}-\mathrm{C}$, Martelletti P, Couturier EGM on behalf of the European Headache Federation and Lifting The Burden: The Global Campaign to Reduce the Burden of Headache Worldwide (2007) European principles of management of common headache disorders in primary care. J Headache Pain 8(Suppl 1):S3-S21 
12. World Health Organization, Lifting The Burden (2011) Atlas of headache disorders and resources in the world 2011. WHO, Geneva

13. Linde M, Gustavsson A, Stovner LJ, Steiner TJ, Barré J, Katsarava Z, Lainez JM, Lampl C, Lantéri-Minet M, Rastenyte D, Ruiz de la Torre E, Tassorelli C, Andrée C (2012) The cost of headache disorders in Europe: the Eurolight project. Eur J Neurol 19:703-711

14. Mennini FS, Gitto L, Martelletti P (2008) Improving care through health economics analyses: cost of illness and headache. J Headache Pain 9: 199-206

15. Linde M, Steiner TJ, Chisholm D (2015) Cost-effectiveness analysis of interventions for migraine in four low- and middle-income countries. Headache Pain 16:15

16. Steiner TJ, Antonaci $F$, Jensen $R$, Lainez JMA, Lantéri-Minet $M$, Valade $D$, on behalf of the European Headache Federation and Lifting The Burden: the Global Campaign against Headache (2011) Recommendations for headache service organisation and delivery in Europe. J Headache Pain 12:419-426

17. Steiner TJ (2004) Lifting the burden: the global campaign against headache. Lancet-Neurol 3:204-205

18. Steiner TJ (2005) Lifting The Burden: the global campaign to reduce the burden of headache worldwide. J Headache Pain 6:373-377

19. Steiner TJ, Birbeck GL, Jensen R, Katsarava Z, Martelletti $P$, Stovner $\sqcup$ (2010) Lifting The Burden: the first 7 years. J Headache Pain 11:451-455

20. Steiner TJ, Birbeck GL, Jensen R, Katsarava Z, Martelletti P, Stovner $\sqcup$ (2011) The global campaign, World Health Organization and Lifting The Burden: collaboration in action. J Headache Pain 12:273-274

21. Buse DC, Sollars CM, Steiner TJ, Jensen RH, Al Jumah MA, Lipton RB (2012) Why HURT? A review of clinical instruments for headache management. Curr Pain Headache Rep 16:237-254

22. Lipton RB, Bigal ME, Diamond M, Freitag F, Reed ML, Stewart WF (2007) Migraine prevalence, disease burden, and the need for preventive therapy. Neurology 68:343-349

23. https://www.bloomberg.com/profiles/companies/9276602Z:US-nfo-inc (accessed 23 December 2017)

24. Lipton RB, Diamond S, Reed M, Diamond ML, Stewart WF (2001) Migraine diagnosis and treatment: results from the American migraine study II. Headache 41:638-645

25. International Headache Society Classification Subcommittee (2004) The international classification of headache disorders. 2nd edition. Cephalalgia 24(Suppl 1):1-160

26. Westergaard ML, Steiner TJ, MacGregor AE, Antonaci F, Tassorelli C, Buse DC, Lipton RB, Jensen RH (2013) The headache under-response to treatment (HURT) questionnaire: assessment of utility in headache specialist care. Cephalalgia 33:245-255

27. Al Jumah M, Al Khathaami A, Tamim H, Al Owayed A, Kojan S, Jawhary A, Lipton R, Buse D, Jensen R, Steiner T (2013) HURT (headache underresponse to treatment) questionnaire in the management of primary headache disorders: reliability, validity and clinical utility of the Arabic version. J Headache Pain 14:6

28. Peters M, Bertolote JM, Houchin C, Kandoura T, Steiner TJ (2007) Translation protocol for lay documents. J Headache Pain 8(Suppl 1):S43-S44

29. Stewart WF, Lipton RB, Kolodner KB, Sawyer J, Lee C, Liberman JN (2000) Validity of the migraine disability assessment (MIDAS) score in comparison to a diary-based measure in a population sample of migraine sufferers. Pain 88:41-52

30. Kosinski M, Bayliss MS, Bjorner JB, Ware JE Jr, Garber WH, Batenhorst A, Cady R, Dahlöf CG, Dowson A, Tepper S (2003) A six-item short-form survey for measuring headache impact: the HIT-6. Qual Life Res 12:963-974

31. Kroenke K, Spitzer RL, Williams JBW (2001) The PHQ-9. Validity of a brief depression severity measure. Gen. Intern Med 16:606-613

32. Martin BC, Pathak DS, Sharfman MI, Adelman JU, Taylor F, Kwong WK, Jhingran P (2000) Validity and reliability of the migraine-specific quality of life questionnaire (MSQ version 2.1). Headache 40:204-215

33. Lipton RB, Serrano D, Buse DC, Rupnow MFT, Reed ML, Bigal ME (2007) The migraine prevention questionnaire (MPQ): development and validation. Headache 47:770-771

34. Stovner LJ, Al Jumah M, Birbeck GL, Gururaj G, Jensen R, Katsarava Z, Queiroz LP, Scher Al, Tekle-Haimanot R, Wang SJ, Steiner TJ (2014) The methodology of population surveys of headache prevalence, burden and cost: principles and recommendations from the global campaign against headache. J Headache Pain 15:5

\section{Submit your manuscript to a SpringerOpen ${ }^{\circ}$ journal and benefit from:}

- Convenient online submission

- Rigorous peer review

- Open access: articles freely available online

- High visibility within the field

- Retaining the copyright to your article

Submit your next manuscript at $\boldsymbol{\nabla}$ springeropen.com 